\title{
Trajectories and risk factors of dementia progression: a memory clinic cohort followed up to 3 years from diagnosis
}

\author{
Trine Holt Edwin, 1,2,3 (D) Bjфrn Heine Strand, 1,3,4 Karin Persson, 1,2 Knut Engedal, 1,2 \\ Geir Selbæk, ${ }^{1,2,3}$ and Anne-Brita Knapskog ${ }^{2}$ \\ ${ }^{1}$ Norwegian National Advisory Unit on Aging and Health, Vestfold Hospital Trust, Tonsberg, Norway \\ ${ }^{2}$ Department of Geriatric Medicine, Oslo University Hospital, Oslo, Norway \\ ${ }^{3}$ Faculty of Medicine, University of Oslo, Oslo, Norway \\ ${ }^{4}$ Norwegian Institute of Public Health, Oslo, Norway
}

Abstract

Objectives: Patients with dementia follow different trajectories of progression. We aimed to investigate which factors at the time of diagnosis could predict trajectory group membership.

Design: Longitudinal observational study.

Setting: Specialized memory clinic, Oslo University Hospital in Norway.

Participants: Patients assessed at the memory clinic, between 12 January 2009 and 31 July 2016, who were registered in the Norwegian Registry of persons assessed for cognitive symptoms (NorCog) and diagnosed with dementia after the baseline examination period $(n=442)$. The patients were followed up to 3 years, with an average of 3.5 examinations.

Measurements: Clinical Dementia Rating Scale Sum of Boxes (CDR-SB), Mini-Mental State Examination (MMSE), the Consortium to Establish a Registry of Alzheimer's disease (CERAD) 10-item word list delayed recall, the Clock Drawing Test, (CDT) Trail Making Test A (TMT-A), and Neuropsychiatric Inventory Questionnaire (NPI-Q). Based on changes in scores on the CDR-SB, we used group-based trajectory modeling (GBTM) to explore the presence of trajectory groups. Multinomial logistic regression was used to explore whether a set of baseline variables could predict trajectory group membership.

Results: Three trajectory groups were identified, one with a slow progression rate and two with more-rapid progression. Rapid progression was associated with older age, lower cognitive function (MMSE and TMT-A), and more-pronounced neuropsychiatric symptoms (NPI-Q) at the time of diagnosis.

Conclusions: Our findings demonstrate the heterogeneity of dementia progression and describe risk factors for rapid progression, emphasizing the need for individual follow-up regimes. For future intervention studies, our results may guide the selection of patients.

Key words: dementia, memory clinics, neuropsychiatric symptoms, risk factors

\section{Introduction}

Dementia disorders progress, leading to disability and, ultimately, death (Strand et al., 2018, 2019). Studies have indicated that subgroups of patients with dementia follow different trajectories of progression, with a large proportion progressing slowly, especially in the earlier stages (Eldholm et al., 2018a; Melis et al., 2019; Song et al., 2018;

Correspondence should be addressed to: Trine Holt Edwin, OUS HF Ullevål sykehus, Postboks 4956 Nydalen, 0424 Oslo, Norway. Phone: + 4748050072. Emails: trine.holt.edwin@gmail.com; trine.edwin@aldringoghelse.no Received 09 Mar 2020; revision requested 05 Jun 2020; revised version received 05 Jul 2020; accepted 10 Aug 2020. First published online 20 Nov 2020
Wang et al., 2019). Several risk factors for rapid progression at the time of diagnosis have been identified, but the findings are conflicting, and different approaches used and risk factors assessed make it challenging to compare results (Melis et al., 2019). Nevertheless, findings have indicated that lower cognitive function, a heavier neuropsychiatric symptoms (NPS) burden, early appearance of extrapyramidal symptoms, and having signs of both Alzheimer's dementia (AD) and dementia with Lewy bodies (DLB) at baseline may predict a rapid decline (Blanc et al., 2017; Song et al., 2018; Tschanz et al., 2011). Regarding age at dementia onset, the results vary, indicating both young and older age at onset as 
predictors of rapid progression (Melis et al., 2019; Song et al., 2018; Tschanz et al., 2011). Progression rate seems to be affected by several factors, but studies have usually examined potential risk factors separately (Melis et al., 2019).

Different methods and assessment scales are used to measure progression, thereby hindering comparison (Melis et al., 2019; Wang et al., 2019). Most studies have used the change in Mini-Mental State Examination (MMSE) or the Clinical Dementia Rating Scale (CDR), while changes in activities of daily living (ADLs), NPS load, and length of time to nursing home admission or to death have been used less frequently (Melis et al., 2019). These outcomes measure different aspects of the dementia syndrome (Eldholm et al., 2018a; Jutkowitz et al., 2017); MMSE might be less sensitive in the earlier as well as the later stages of dementia (Musicco et al., 2010; Song et al., 2018), while the CDR, measuring both cognitive and functional abilities (Hughes et al., 1982), is more appropriate for detecting changes during all stages. The statistical methods applied to investigate dementia progression also vary, complicating the comparison of studies even further (Melis et al., 2019).

Identifying risk factors for progression and understanding the course of dementia are important for more-precise prognoses. This is essential in the care of patients, for informing policymakers, and in the search for effective disease-modifying treatments (Shah et al., 2016). By identifying distinct trajectory groups, we study the heterogeneity of dementia progression in a Norwegian memory clinic cohort. We further investigate whether a set of baseline factors could predict trajectory group membership.

\section{Methods}

\section{Participants}

Patients assessed at the memory clinic, Oslo University Hospital, between 12 January 2009 and 31 July 2016, who were registered in the Norwegian Registry of persons assessed for cognitive symptoms (NorCog) and diagnosed with dementia after the baseline examination period (defined as up to 6 months from the first visit) (number of patients $[n]=668$ ), were eligible for inclusion (see Figure S1 published as supplementary material online attached to the electronic version of this paper). Patients who did not receive any follow-up examinations at the memory clinic were excluded $(n=219)$. Patients received follow-up at various time intervals based on clinical judgment. The CDR was scored as a measure of cognitive and functional impairment (Hughes et al., 1982) at each clinic visit (see section: Main study outcome), and in the current study, patients were followed up to 3 years after diagnosis. Maximum follow-up was restricted to 3 years due to limited data after that period and to limit survival bias. During this time window, the patients received an average of 3.5 (standard deviation [SD] 1.7) examinations, including the baseline examination. Seven patients were excluded due to insufficient information to assess the CDR on at least two occasions.

All patients gave written informed consent to participate. The present project was approved by the Regional Ethics Committee (2015/1510 REK vest) and was performed in accordance with the Helsinki Declaration of 1975.

\section{Diagnostic workup}

All patients were assessed at baseline according to the standardized and comprehensive NorCog research protocol (Braekhus et al., 2011) as a part of a routine clinical workup. This includes a cognitive test battery, detailed information about symptoms, number of years of education, medication use, and number of comorbid diagnoses within the following disease categories: pulmonary, cerebrovascular, cardiovascular, cancer, or diabetes mellitus (registered in a standardized way by a physician). All patients underwent a physical examination including blood sampling and, in most cases, a magnetic resonance imaging brain scan (MRI). According to clinical indication, cerebrospinal fluid core biomarkers $(n=198)$ amyloid $\beta 42$, total tau, and phosphorylated ${ }^{181}$ tau were measured, and positron emission tomography or single-photon emission computed tomography was performed (Braekhus et al., 2011). These biomarkers were used in the diagnostic process. We included the following cognitive tests as predictors of progression rate: the MMSE (0-30; lower values indicate greater cognitive impairment) (Folstein et al., 1975); the Consortium to Establish a Registry of Alzheimer's Disease (CERAD) 10-item word list delayed recall (CERAD-DR) (0-10; lower values indicate poorer episodic memory function) (Morris et al., 1989); the Clock Drawing Test (CDT) (Mainland et al., 2014) (with pathological cutoff $\leq 3 / 5$ points [Shulman et al., 1986]); and the Trail Making Test A (TMT-A) (Reitan, 1958) (based on age-adjusted cutoff of -2 SD [Mitrushina et al., 2005]). NPS were assessed using the Neuropsychiatric Inventory Questionnaire (NPI-Q) (Kaufer et al., 2000), a proxy-based short form of the Neuropsychiatric Inventory (NPI) (Cummings et al., 1994), and the NPI-Q severity score (0-36; higher values indicate more-severe NPS burden) was included in the analyses.

Based on all available information from the baseline examinations, diagnoses were made by one of the researchers who also is an experienced clinician. 
In inconclusive cases $(n=61)$, two of the other researchers who also are experienced clinicians were consulted. The National Institute on Aging and the Alzheimer's Association (NIA-AA) diagnostic criteria were used to diagnose all-cause dementia (McKhann et al., 2011). AD and etiologically mixed $\mathrm{AD}$ ( $\mathrm{AD}$ mixed) were diagnosed according to the NIA-AA criteria for Alzheimer's disease dementia (McKhann et al., 2011). Parkinson's disease dementia (PDD) and DLB were diagnosed using the clinical diagnostic criteria for dementia associated with Parkinson's disease (Emre et al., 2007) and the revised criteria from the fourth consensus report of the DLB Consortium (McKeith et al., 2017), respectively. Frontotemporal dementia was diagnosed using the International Consortium Revised Guidelines for the diagnosis of behavioral-variant frontotemporal dementia (bv-FTD) (Rascovsky et al., 2011) and classification of primary progressive aphasia (PPA) (Gorno-Tempini et al., 2011). If none of these were present, the etiology was denoted as dementia caused by other etiology (other).

\section{Main study outcome: CDR}

As a global measure of cognitive and functional impairment, the CDR (Hughes et al., 1982) was scored by the same researcher who is a certified CDR rater (Knight ADRC) based on all information from the patient's record at baseline and at every follow-up examination. In equivocal cases, two additional researchers who are experienced CDR raters were consulted. The CDR rates a person's cognitive ability in relation to past performance in six cognitive and functional categories: memory, orientation, judgment and problem-solving, community affairs, home and hobbies, and personal care. Each item was given a score of $0,0.5,1,2$, or 3 according to the severity of the decline. The global score is based on an algorithm that gives priority to the memory item, ranging from CDR 0 (no dementia) to CDR 3 (severe dementia) (Hughes et al., 1982). In research, the items are often added together as the CDR sum of boxes (CDR-SB) to form a continuous scale $(0-18$, higher scores indicate more-severe decline) (O'Bryant et al., 2008).

\section{Statistical analyses}

Analyses were performed using Stata/IC 15.1 (StataCorp LLC 2018, Stata Statistical Software, revision 17 December 2018, College Station, TX, USA). To compare the difference in baseline characteristics and mortality between groups, independent $t$-tests and Pearson's $\chi^{2}$ tests were used for continuous and categorical data, respectively. For participants who died during the 3 years of follow-up, date of death was linked to the cohort using the Cause of Death Registry (Norwegian Institute of Public Health, 2018). To explore the presence of distinct trajectory groups based on the change in the CDR-SB over time, we first applied group-based trajectory modeling (GBTM) (Nagin and Odgers, 2010), using the Stata package Traj (Jones and Nagin, 2013). The number and shapes of trajectory groups was decided guided by model fit as well as clinical relevance, as suggested by Nagin and Odgers, ensuring sufficient class size and clinical usefulness (Nagin and Odgers, 2010). More specifically, we used the Bayesian information criterion (BIC) to estimate the goodness-of-fit of the different models; values closest to zero indicate better fit. Furthermore, we ensured the posterior probability of group membership to be at least 0.7 and odds of correct classification (OCC) to be above 5 (see Table S1 published as supplementary material online attached to the electronic version of this paper). We also checked that the confidence intervals (CIs) of the trajectory groups did not overlap, indicating a good model fit (Nagin and Odgers, 2010).

Next, the three-level trajectory group membership categorical variable was applied as the outcome variable in multinomial logistic regression to explore whether a set of baseline characteristics could predict trajectory group membership. We ensured that Spearman's intercorrelations between the explanatory variables were not $\geq 0.5$, and thus, the TMT-B was removed due to high intercorrelation with the TMT-A. A set of regression models was fitted, all adjusted for sex, age, years of education, dementia etiology, and comorbidity (model 1), by separately including the variables of interest: MMSE (model 2), NPI-Q (model 3), TMT-A (model 4), CDT (model 5), and CERAD-DR (model 6). In the final model, all variables were included (model 7). Only those with a complete set of baseline variables were included in the regression analyses $(n=372)$. The fit of the different models was assessed using likelihoodratio tests for nested models.

\section{Results}

\section{Sample characteristics}

Mean age at diagnosis was 70.5 years (range 46-89 years); $51 \%$ were females (Table 1 ). Most patients had $\mathrm{AD}$ (AD or AD mixed) (73\%). Mean MMSE at baseline was 23.1 (SD 4.1), mean CDR-SB was 5.1 (SD 2.3), and most patients $(54 \%)$ did not have any registered comorbidity.

Those without follow-up examinations were older, and fewer were living with a partner. In addition, they had lower baseline cognitive functioning, more-severe 
Table 1. Descriptive statistics of patients who did and did not receive follow-up examinations

\begin{tabular}{|c|c|c|c|}
\hline & WITH FOLLOW-UP & WITHOUT FOLLOW-UP & \\
\hline CHARACTERISTICS & $(N=442)$ & $(N=219)$ & $P$-VALUE \\
\hline Age, years & $70.5(8.1)$ & $73.6(9.4)$ & $<0.001$ \\
\hline Young onset, $<65$ years, N (\%) & $124(28.1)$ & $48(21.9)$ & 0.091 \\
\hline Female, $\mathrm{N}(\%)$ & $225(50.9)$ & $104(47.5)$ & 0.408 \\
\hline Married/living together with partner, $\mathrm{N}(\%)$ & $323(73.1)$ & $137(62.6)$ & 0.006 \\
\hline Years of education & $12.8(3.7)$ & $12.3(3.8)$ & 0.141 \\
\hline \multicolumn{4}{|l|}{ Dementia etiology, $\mathrm{N}(\%)$} \\
\hline $\mathrm{AD}$ & $229(51.8)$ & $90(41.1)$ & 0.009 \\
\hline $\mathrm{AD}$ mixed & $93(21.0)$ & $51(23.3)$ & 0.510 \\
\hline $\mathrm{DLB} / \mathrm{PDD}$ & $49(11.1)$ & $29(13.3)$ & 0.419 \\
\hline FTD & $29(6.6)$ & $16(7.3)$ & 0.720 \\
\hline Other & $42(9.5)$ & $33(15.1)$ & 0.034 \\
\hline Comorbidity present ( $\geq 1$ disease), $\mathrm{N}(\%)$ & $195(46)$ & $98(46)$ & 0.935 \\
\hline Pulmonary disease, $\mathrm{N}(\%)$ & $5(1.1)$ & $6(2.8)$ & 0.128 \\
\hline Cerebrovascular disease, $\mathrm{N}(\%)$ & $57(12.9)$ & $44(20.1)$ & 0.016 \\
\hline Cardiovascular disease, $\mathrm{N}(\%)$ & $137(31.0)$ & $73(33.3)$ & 0.543 \\
\hline Cancer, $\mathrm{N}(\%)$ & $42(9.5)$ & $11(5.0)$ & 0.046 \\
\hline Diabetes mellitus, N (\%) & $38(8.6)$ & $19(8.6)$ & 0.973 \\
\hline MMSE & $23.1(4.1)$ & $21.6(4.4)$ & $<0.001$ \\
\hline NPI-Q symptoms & $3.7(2.6)$ & $4.5(2.6)$ & 0.001 \\
\hline NPI-Q severity & $5.8(5.3)$ & $7.2(5.6)$ & 0.002 \\
\hline TMT-A (worse than - 2 SD), N (\%) & $206(48.1)$ & $127(62.3)$ & 0.001 \\
\hline $\operatorname{CDT}(\leq 3 / 5$ points $), \mathrm{N}(\%)$ & $259(58.9)$ & $148(69.5)$ & 0.009 \\
\hline CERAD-DR & $1.6(1.9)$ & $1.7(1.9)$ & 0.549 \\
\hline Follow-up, years & $2.2(1.5)$ & & $*$ \\
\hline CDR sum of boxes & $5.1(2.3)$ & $7.1(3.1)$ & $<0.001$ \\
\hline CDR sum of boxes yearly change (mean, SE) & $0.9 \pm 0.06$ & & $*$ \\
\hline $\begin{array}{l}\text { Mortality the first } 3 \text { years after the diagnosis } \\
\text { (per } 1000 \text { person-years) }\end{array}$ & 29.2 & 95.8 & $<0.001$ \\
\hline
\end{tabular}

Note. The values are presented as means (standard deviation, SD) unless otherwise specified. The descriptive means of the groups were compared using independent $t$-tests, and proportions were compared using Pearson's $\chi^{2}$ tests. Bold values highlight significant differences $(p \leq 0.05)$.

Abbreviations: N, number of patients; SD, standard deviation; SE, standard error; AD, Alzheimer's dementia; AD mixed, etiologically mixed Alzheimer's dementia; DLB, dementia with Lewy bodies; PDD, Parkinson's disease dementia; FTD, frontotemporal dementia; MMSE, Mini-Mental State Examination; NPI-Q, Neuropsychiatric Inventory Questionnaire; TMT-A, Trail Making Test A; CDT, the Clock Drawing Test; CERAD-DR, the Consortium to Establish a Registry of Alzheimer's Disease 10-item word list delayed recall; CDR, Clinical Dementia Rating Scale.

*Not applicable.

NPS burden, more-severe dementia, and a higher mortality compared to those with follow-up examinations (Table 1). Number of comorbidities, were similar between the groups, but in the group who was not followed up, more had cerebrovascular disease $(p=0.016)$ and less had cancer $(p=0.046)$ (Table 1).

\section{Trajectory groups}

Three distinct progression-trajectory groups were identified (Figure 1). Group 1 had the best baseline functioning and the slowest progression rate (average baseline CDR-SB $=3.5$ and average annual change in CDR-SB $=0.7)$. Groups 2 and 3 both progressed faster (annual change in
$\mathrm{CDR}-\mathrm{SB}=2.4$ and 2.9 , respectively), and their baseline level of dementia differed, with group 3 having more-advanced impairment (CDR$\mathrm{SB}=5.3$ and 8.2, respectively) (Table 2).

During follow-up, mortality (per 1000 personyears) for the three groups were 17.0 (95\% CI 8.2, 32.6), 19.6 (95\% CI 9.8, 39.3), and 70.6 (95\% CI $44.4,112.0$ ), respectively. Thus, patients in group 3 had more than 4 times the mortality of those in group 1 (Table 2). No differences were found in the number of patients with pulmonary disease $(p=0.968)$, cerebrovascular disease $(p=0.899)$, cardiovascular disease $(p=0.535)$, or cancer $(p=0.104)$ between the trajectory groups. There were, however, more patients with diabetes mellitus in the rapidly progressing group $(p=0.005)$. 


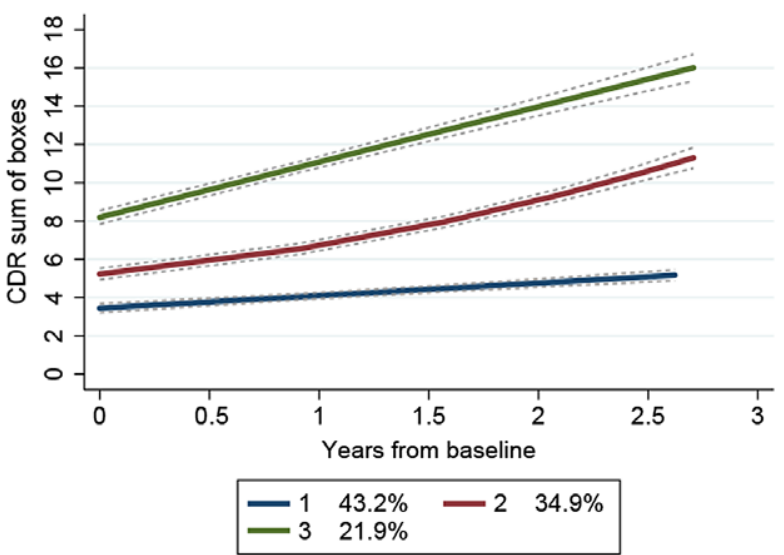

Figure 1. Three trajectory groups based on change in CDR sum of boxes over time. Note. Trajectory groups, using GBTM, with the trajectory shapes 121 ( 1 = linear, 2 = quadratic). The proportions (\%) are based on the maximum probability assignment rule. Group 1 (blue); number of patients $(n)=195(43.2 \%)$, posterior probability of group membership $=0.92$, and OCC $=14.7$. Group 2 (red); $n=153(34.9 \%)$ posterior probability of group membership = 0.88 , and OCC $=13.4$. Group 3 (green); $n=94(21.9 \%)$, posterior probability of group membership $=0.94$, and $O C C=62.6$. CDR, clinical dementia rating scale. Production: File format TIFF, using Stata/IC 15.1 StataCorp LLC 2018 on Windows 10 Pro.

\section{Predictors of rapid progression}

\section{BaSELINE FACTORS ASSOCIATED With}

TRAJECTORY GROUP 2 MEMBERSHIP (MORE-RAPID PROGRESSION)

In the fully adjusted model (Table 4), using group 1 as a reference, we found that belonging to group 2 was associated with higher age (relative risk ratio [RRR] 1.04 [95\% CI 1.00, 1.07]), lower score on the MMSE (RRR 0.86 [95\% CI $0.79,0.94]$ ), and worse performance on the TMT-A (RRR 0.35 [95\% CI $0.20,0.61]$ ) at the baseline examination. For every single-point increase in severity on the NPI-Q, the risk of belonging to group 2 increased by $7 \%$ (RRR 1.07 [95\% CI 1.02, 1.13]). Further, belonging to group 2 was associated with poor performance on the CDT in the crude model (RRR 0.45 [95\% CI $0.27,0.73]$ ) (Table 3), but this was no longer significant in the fully adjusted model. No significant differences were found in regard to sex, years of education, dementia etiology, comorbidity, or performance on CERAD-DR between group 1 and the more-rapidly progressing group 2 in either model.

\section{BASELINE FACTORS ASSOCIATED WITH} TRAJECTORY GROUP 3 MEMBERSHIP (MOSTRAPIDLY PROGRESSING GROUP) In the fully adjusted model (Table 4 ), using group 1 as a reference, we found that belonging to group 3 was associated with higher age (RRR $1.06[95 \% \mathrm{CI}$
$1.01,1.11])$, lower score on the MMSE (RRR 0.68 [95\% CI 0.61,0.77]), and worse performance on the TMT-A (RRR 0.39 [95\% CI 0.18, 0.84]) at the baseline examination. For every single-point increase in severity on the NPI-Q, the risk of belonging to group 3 increased by $16 \%$ (RRR 1.16 [95\% CI 1.09, 1.24]). Belonging to group 3 was associated with less education (model 1) (RRR 0.92 [95\% CI 0.85, 0.99]), poor performance on the CDT (model 5) (RRR 0.26 [95\% CI 0.14, $0.48]$ ), and on the CERAD-DR (model 6) (RRR 0.77 [95\% CI $0.63,0.93]$ ) in the crude models (Table 3), but these were no longer significant in the fully adjusted model. There were no significant differences in sex, dementia etiology, or comorbidity between the groups (group 1 vs. group 3) in either model.

WHICH BASELINE PREDICTORS WERE MORE IMPORTANT FOR PREDICTING TRAJECTORY GROUP MEMBERSHIP?

The MMSE $(p<0.001)$, NPI-Q severity score $(p<0.001)$, and TMT-A $(p=0.001)$ were the most important single predictors of dementia progression.

\section{Discussion}

In a sample of patients with dementia examined at a specialized memory clinic in Norway and followed up to 3 years after diagnosis, we found three distinct trajectory groups of dementia progression; one group progressed slowly, while two other groups had a more-rapid decline. Rapid progression was associated with older age, lower cognitive function, and more-pronounced NPS at the time of diagnosis.

Our findings are in agreement with those of other studies showing heterogeneity in the progression rate of patients with dementia (Eldholm et al., 2018a; Haaksma et al., 2018; Melis et al., 2019; Song et al., 2018; Wang et al., 2019). The trajectory groups in our sample differed regarding baseline cognitive and functional abilities, indicating the patients were at different stages of dementia at baseline. A meta-analysis from 2018 found that moderate dementia, defined as a score on the MMSE between 17 and 21, was associated with rapid cognitive decline, defined as a yearly change in MMSE $\geq 3$ points (follow-up time ranging from 61.2 days to 9 years) (Song et al., 2018). This could indicate that cognitive decline reaches a threshold, after which it accelerates and then subsequently slows in severe dementia when there is less cognitive function to be lost (Musicco et al., 2010; Song et al., 2018). We did, however, find significant baseline 
Table 2. Descriptive statistics of the three trajectory groups

\begin{tabular}{|c|c|c|c|}
\hline & GROUP 1 & GROUP 2 & GROUP 3 \\
\hline CHARACTERISTICS & $(N=195)$ & $(N=153)$ & $(N=94)$ \\
\hline Age, years & $69.7(7.9)$ & $71.3(7.6)$ & $70.7(9.0)$ \\
\hline Young onset, $<65$ years, N (\%) & $64(32.8)$ & $34(22.2)$ & $26(27.7)$ \\
\hline Female, N (\%) & $89(45.6)$ & $84(54.9)$ & $53(55.3)$ \\
\hline Married/living together with partner, N (\%) & $151(77.4)$ & $106(69.3)$ & $66(70.2)$ \\
\hline Years of education & $13.2(3.6)$ & $12.8(3.7)$ & $12.0(3.9)$ \\
\hline \multicolumn{4}{|l|}{ Dementia etiology, $\mathrm{N}(\%)$} \\
\hline $\mathrm{AD}$ & $106(54.4)$ & $72(47.1)$ & $51(54.3)$ \\
\hline $\mathrm{AD}$ mixed & $33(16.9)$ & $36(23.5)$ & $24(25.5)$ \\
\hline $\mathrm{DLB} / \mathrm{PDD}$ & $19(9.7)$ & $22(14.4)$ & $8(8.5)$ \\
\hline FTD & $12(6.2)$ & $9(5.9)$ & $8(8.5)$ \\
\hline Other & $25(12.8)$ & $14(9.2)$ & $3(3.2)$ \\
\hline Comorbidity present ( $\geq 1$ disease), $\mathrm{N}(\%)$ & $93(49.2)$ & $67(46.3)$ & $34(36.6)$ \\
\hline Pulmonary disease, $\mathrm{N}(\%)$ & $2(1.0)$ & $2(1.3)$ & $1(1.1)$ \\
\hline Cerebrovascular disease, $\mathrm{N}(\%)$ & $25(12.8)$ & $21(13.7)$ & $11(11.7)$ \\
\hline Cardiovascular disease, $\mathrm{N}(\%)$ & $61(31.3)$ & $51(33.3)$ & $25(26.6)$ \\
\hline Cancer, $\mathrm{N}(\%)$ & $25(12.8)$ & $10(6.5)$ & $7(4.5)$ \\
\hline Diabetes mellitus, N (\%) & $9(4.6)$ & $14(9.2)$ & $15(16.0)$ \\
\hline MMSE & $24.9(2.8)$ & $23.0(3.4)$ & $19.5(5.0)$ \\
\hline NPI-Q symptoms & $3.0(2.4)$ & $3.8(2.5)$ & $4.9(2.6)$ \\
\hline NPI-Q severity & $4.5(4.6)$ & $5.9(5.0)$ & $8.3(6.0)$ \\
\hline TMT-A (worse than -2 SD), N (\%) & $63(33.0)$ & $88(58.7)$ & $55(63.2)$ \\
\hline $\mathrm{CDT}(\leq 3 / 5$ points $), \mathrm{N}(\%)$ & $88(45.6)$ & $99(64.7)$ & $72(76.6)$ \\
\hline CERAD-DR & $1.9(2.0)$ & $1.5(1.8)$ & $0.9(1.3)$ \\
\hline CDR sum of boxes & $3.5(1.0)$ & $5.3(1.4)$ & $8.2(2.2)$ \\
\hline CDR sum of boxes yearly change (mean, SE) & $0.7(0.1)$ & $2.4(0.6)$ & $2.9(0.2)$ \\
\hline Mortality the first 3 years after the diagnosis (per 1000 person-years) & 17.0 & 19.6 & 70.6 \\
\hline
\end{tabular}

Note. The values are presented as means (standard deviation, SD) unless otherwise specified.

Abbreviations: N, number of patients; SD, standard deviation; SE, standard error; AD, Alzheimer's dementia; AD mixed, etiologically mixed Alzheimer's dementia; DLB, dementia with Lewy bodies; PDD, Parkinson's disease dementia; FTD, frontotemporal dementia; MMSE, Mini-Mental State Examination; NPI-Q, Neuropsychiatric Inventory Questionnaire; TMT-A, Trail Making Test A; CDT, the Clock Drawing Test; CERAD-DR, the Consortium to Establish a Registry of Alzheimer's Disease 10-item word list delayed recall; CDR, Clinical Dementia Rating Scale.

differences between the trajectory groups, even after adjusting for cognition, indicating that the groups do reflect different trajectories of progression. Like us, Haaksma et al. examined the progression of dementia using the CDR-SB over 3 years in a large clinical cohort. They also found three distinct trajectory groups, with a large group (63\%) progressing slowly. Their study differed from ours in several aspects; only $\mathrm{AD}$ patients were included; participants were older ( 79.4 vs. 70.5 years), baseline cognitive and functional abilities were better (CDR-SB 3.8 vs. 5.1), and the methods differed (Haaksma et al., 2018). Still, our similar results strengthen the belief that these three trajectory groups are clinically relevant.

In our sample, we found that older age at baseline could predict a more-rapid progression rate. In the literature, the impact of age at onset has been inconclusive indicating that onset at both a younger and older age could predict rapid progression (Haaksma et al., 2018; Lanctot et al., 2017; Melis et al., 2019;
Song et al., 2018). This discrepancy might be due to different methods used or to differences in patient characteristics. Even though we did not find any significant differences in the number of baseline comorbidities between the groups, older patients are at risk of concomitant incidents and frailty, and this is suggested to have at least a short-term impact on progression rate (Haaksma et al., 2017). Older patients also have an increased risk of experiencing delirium during the follow-up period (Vasilevskis et al., 2012), and delirium superimposed on dementia has been shown to accelerate cognitive decline (Krogseth et al., 2016). According to the Norwegian guidelines on dementia, specialist healthcare services are responsible for assessing patients with cognitive symptoms who are younger (guiding recommendation of $\leq 65$ years), experiencing atypical symptoms or having other complicating factors (Norwegian Directorate of Health, 2017). Therefore, our older patients might have had a more atypical presentation, which has 
Table 3. Multinomial logistic regression small models (models 1-6) assessing trajectory group membership by baseline predictors

\begin{tabular}{|c|c|c|c|c|c|c|}
\hline \multirow{2}{*}{$\begin{array}{l}\text { SMALL MODELS } 1-6(N=372) \\
\text { Characteristics }\end{array}$} & \multicolumn{3}{|c|}{ GROUP 2 VERSUS GROUP 1} & \multicolumn{3}{|c|}{ GROUP 3 VERSUS GROUP 1} \\
\hline & RRR & $95 \% \mathrm{CI}$ & $p$-value & RRR & $95 \% \mathrm{CI}$ & $p$-value \\
\hline \multicolumn{7}{|l|}{ Sex $^{*}$} \\
\hline Female & 1.0 & & & 1.0 & & \\
\hline Male & 0.72 & $(0.47,1.40)$ & 0.398 & 0.97 & $(0.52,1.82)$ & 0.927 \\
\hline $\operatorname{Age}^{*}$ & 1.03 & $(1.00,1.06)$ & 0.127 & 1.03 & $(1.00,1.07)$ & 0.069 \\
\hline Years of education ${ }^{*}$ & 0.98 & $(0.92,1.05)$ & 0.653 & 0.92 & $(0.85,0.99)$ & 0.037 \\
\hline \multicolumn{7}{|l|}{ Dementia etiology $^{*}$} \\
\hline $\mathrm{AD}$ & 1.0 & & & 1.0 & & \\
\hline $\mathrm{AD}$ mixed & 1.50 & $(0.80,2.78)$ & 0.207 & 1.56 & $(0.77,3.15)$ & 0.218 \\
\hline $\mathrm{DLB} / \mathrm{PDD}$ & 1.63 & $(0.80,3.32)$ & 0.175 & 0.88 & $(0.35,2.24)$ & 0.787 \\
\hline FTD & 1.34 & $(0.49,3.75)$ & 0.552 & 1.21 & $(0.34,4.27)$ & 0.764 \\
\hline Other & 0.54 & $(0.20,1.48)$ & 0.235 & 0.49 & $(0.13,1.83)$ & 0.293 \\
\hline \multicolumn{7}{|l|}{ Comorbidity $^{*}$} \\
\hline No comorbidity & 1.0 & & & 1.0 & & \\
\hline$\geq 1$ disease & 0.86 & $(0.53,1.40)$ & 0.550 & 0.68 & $(0.38,1.20)$ & 0.180 \\
\hline $\mathrm{MMSE}^{\dagger}$ & 0.82 & $(0.76,0.89)$ & $<0.001$ & 0.66 & $(0.59,0.73)$ & $<0.001$ \\
\hline NPI severity ${ }^{\ddagger}$ & 1.08 & $(1.02,1.13)$ & 0.006 & 1.15 & $(1.09,1.22)$ & $<0.001$ \\
\hline \multicolumn{7}{|l|}{ TMT-A } \\
\hline$-2 \mathrm{SD}$ or worse & 1.0 & & & 1.0 & & \\
\hline Better than -2 SD & 0.27 & $(0.16,0.45)$ & $<0.001$ & 0.19 & $(0.10,0.35)$ & $<0.001$ \\
\hline \multicolumn{7}{|l|}{$\mathrm{CDT}^{\pi}$} \\
\hline$\leq 3 / 5$ points & 1.0 & & & 1.0 & & \\
\hline$\geq 4 / 5$ points & 0.45 & $(0.27,0.73)$ & 0.001 & 0.26 & $(0.14,0.48)$ & $<0.001$ \\
\hline CERAD-DR\# & 0.95 & $(0.83,1.10)$ & 0.519 & 0.77 & $(0.63,0.93)$ & 0.008 \\
\hline
\end{tabular}

Note. Multinomial logistic regression models (models 1-6) were used to assess predictors of trajectory group membership, by separately testing baseline covariates of interest. All models were adjusted by sex, age, years of education, dementia etiology, and comorbidity. Bold values highlight significant differences $(p \leq 0.05)$.

Abbreviations: N, number of patients; SD, standard deviation; RRR, relative risk ratio; CI, confidence interval; AD, Alzheimer's dementia; AD mixed, etiologically mixed Alzheimer's dementia; DLB, dementia with Lewy bodies; PDD, Parkinson's disease dementia; FTD, frontotemporal dementia; MMSE, Mini-Mental State Examination; NPI-Q, Neuropsychiatric Inventory Questionnaire; TMT-A, Trail Making Test A; CDT, the Clock Drawing Test; CERAD-DR, the Consortium to Establish a Registry of Alzheimer's Disease 10-item word list delayed recall.

* Model 1.

†Model 2.

$¥$ Model 3.

$\$$ Model 4.

ๆModel 5.

\# Model 6.

been shown to predict rapid decline (Scheltens et al., 2018). Our findings also suggest cognitive functions such as psychomotor retardation, but not memory loss could predict a rapid decline. This supports the belief that non-memory deficits are a sign of a moreaggressive disease process, at least in $\mathrm{AD}$ (Scheltens et al., 2018).

Rapid progression of dementia was associated with more-severe NPS load at baseline, after adjusting for cognitive test results, age, sex, education, dementia etiology, and comorbidity. Other studies have also indicated NPS burden as a predictor of accelerated cognitive and functional decline (Lanctot et al., 2017; Poulin et al., 2017; Song et al., 2018), and increased risk of early institutionalization, poor quality of life and mortality (Lanctot et al., 2017). Moreover, NPS has been shown to increase caregiver burden (Reed et al., 2020). Even though NPS can occur at all stages of dementia, the severity has been shown to increase in the more-advanced stages (Jutkowitz et al., 2017; Lanctot et al., 2017; Lyketsos et al., 2002; Poulin et al., 2017). This could reflect that NPS is a result of more-pronounced neurodegeneration affecting the regulation of behavior (Lanctot et al., 2017). However, since the progression of NPS and cognitive decline seem to follow different trajectories, and since NPS is a risk factor for progression even after adjusting for cognitive decline, we believe it is a poor prognostic factor in dementia (Poulin et al., 2017; Tschanz et al., 2011). In the present study, we did not study the individual NPS, although the symptoms probably have different underlying pathologies and the frequency may vary across dementia severity 
Table 4. Fully adjusted multinomial logistic regression model (model 7), assessing trajectory group membership by baseline predictors

\begin{tabular}{|c|c|c|c|c|c|c|}
\hline \multirow{2}{*}{$\begin{array}{l}\text { MODEL } 7(N=372) \\
\text { Characteristics }\end{array}$} & \multicolumn{3}{|c|}{ GROUP 2 VERSUS GROUP 1} & \multicolumn{3}{|c|}{ GROUP 3 VERSUS GROUP 1} \\
\hline & RRR & $95 \% \mathrm{CI}$ & $p$-value & RRR & $95 \% \mathrm{CI}$ & $p$-value \\
\hline \multicolumn{7}{|l|}{ Sex } \\
\hline Female & 1.0 & & & 1.0 & & \\
\hline Male & 0.91 & $(0.52,1.59)$ & 0.737 & 1.34 & $(0.64,2.81)$ & 0.442 \\
\hline Age & 1.04 & $(1.00,1.07)$ & 0.028 & 1.06 & $(1.01,1.11)$ & 0.013 \\
\hline Years of education & 1.04 & $(0.96,1.12)$ & 0.337 & 1.03 & $(0.94,1.13)$ & 0.559 \\
\hline \multicolumn{7}{|l|}{ Dementia etiology } \\
\hline $\mathrm{AD}$ & 1.00 & & & 1.00 & & \\
\hline $\mathrm{AD}$ mixed & 1.65 & $(0.84,3.23)$ & 0.144 & 2.27 & $(0.96,5.38)$ & 0.062 \\
\hline $\mathrm{DLB} / \mathrm{PDD}$ & 1.21 & $(0.54,2.71)$ & 0.650 & 0.79 & $(0.24,2.59)$ & 0.693 \\
\hline FTD & 1.59 & $(0.54,4.72)$ & 0.404 & 1.29 & $(0.25,6.67)$ & 0.765 \\
\hline Other & 0.54 & $(0.18,1.62)$ & 0.272 & 1.18 & $(0.26,5.35)$ & 0.826 \\
\hline \multicolumn{7}{|l|}{ Comorbidity } \\
\hline No comorbidity & 1.00 & & & 1.00 & & \\
\hline$\geq 1$ disease & 1.07 & $(0.63,1.80)$ & 0.809 & 0.93 & $(0.46,1.87)$ & 0.833 \\
\hline MMSE & 0.86 & $(0.79,0.94)$ & 0.001 & 0.68 & $(0.61,0.77)$ & $<0.001$ \\
\hline NPI-Q severity & 1.07 & $(1.02,1.13)$ & 0.010 & 1.16 & $(1.09,1.24)$ & $<0.001$ \\
\hline \multicolumn{7}{|l|}{ TMT-A } \\
\hline$-2 \mathrm{SD}$ or worse & 1.00 & & & 1.00 & & \\
\hline Better than -2 SD & 0.35 & $(0.20,0.61)$ & $<0.001$ & 0.39 & $(0.18,0.84)$ & 0.016 \\
\hline \multicolumn{7}{|l|}{ CDT } \\
\hline$\leq 3 / 5$ points & 1.00 & & & 1.00 & & \\
\hline$\geq 4 / 5$ points & 0.80 & $(0.46,1.40)$ & 0.439 & 0.90 & $(0.41,1.95)$ & 0.787 \\
\hline CERAD-DR & 0.98 & $(0.84,1.15)$ & 0.817 & 0.92 & $(0.72,1.18)$ & 0.526 \\
\hline
\end{tabular}

Note. A fully adjusted multinomial logistic regression model was used to assess predictors of trajectory group membership by baseline covariates. Bold values highlight significant differences $(P \leq 0.05)$.

Abbreviations: N, number of patients; SD, standard deviation; RRR, relative risk ratio; CI, confidence interval; AD, Alzheimer's dementia; $\mathrm{AD}$ mixed, etiologically mixed Alzheimer's dementia; DLB, dementia with Lewy bodies; PDD, Parkinson's disease dementia; FTD, frontotemporal dementia; MMSE, Mini-Mental State Examination; NPI-Q, Neuropsychiatric Inventory Questionnaire; TMT-A, Trail Making Test A; CDT, the Clock Drawing Test; CERAD-DR, the Consortium to Establish a Registry of Alzheimer's Disease 10-item word list delayed recall.

(Siafarikas et al., 2018). The effect of individual NPS on dementia progression should, therefore, be investigated further. It is also unclear whether interventions in regard to NPS will affect the progression rate (Lanctot et al., 2017).

Among those being followed, the rapid progressors had the highest mortality (Table 2), but those without follow-up examinations had even higher mortality and more NPS (Table 1). More advanced dementia or possibly the use of antipsychotics (Schneider et al., 2005) could have contributed to this increased mortality. Those without follow-up had more cardiovascular disease $(p=0.016)$ which could have contributed to increased mortality in this group. On the contrary, they had less cancer, but our data do not provide information about the type and severity of the malignancy. There were no differences in the amount of cardiovascular disease between the trajectory groups $(p=0.535)$. Interestingly, more of the rapid progressors had diabetes mellitus, which may have contributed to higher mortality (Xu et al., 2018), but which did not increase the risk of rapid dementia progression in a Systematic Review and Meta-Analysis (Song et al., 2018). Comorbid diseases probably have different effects on the progression rate of dementia, and this deserves further attention.

There were no significant differences in progression between the various dementia etiologies in our sample, but there was a trend toward higher risk of belonging to group 3 with the diagnosis $\mathrm{AD}$ mixed compared to AD alone (RRR 2.27 [ 95\% CI 0.96, 5.38] $p=0.062$ ). A study by Blanc et al. showed that having both AD and DLB together could predict a more-rapid progression compared to having either one alone (Blanc et al., 2017). The progression was, however, measured by change in the MMSE, which is not equivalent to change in the CDR-SB since the CDR considers both cognitive and functional abilities (Hughes et al., 1982). In a study by Eldholm et al., no association was found between progression rate (measured by change in the CDR-SB) and vascular comorbidity in patients with $\mathrm{AD}$ (Eldholm et al., 2018b). Since most of our patients with a 
diagnosis of $\mathrm{AD}$ mixed had cerebrovascular disease as the concomitant condition (92\%), this might explain why we did not find a significant result. A study by Strand et al., from the NorCog Registry, found differences in reduced life expectancy between the various causes of dementia, with the greatest loss in patients with vascular dementia, DLB, and PDD (Strand et al., 2018). Decline in the CDR and reduced life expectancy can both be used to indicate the prognosis of a patient, but they are also not entirely comparable. In addition, as most of the patients in the present study were affected by $\mathrm{AD}$ or $\mathrm{AD}$ mixed presentation $(73 \%)$, we might not have had sufficient power to show a potential difference within the non-AD populations.

Higher education has previously been shown to predict a more-rapid decline (Haaksma et al., 2018; Song et al., 2018), but we found no such association.

A limitation in our study is that the study population is a selected group who were referred to a specialized memory clinic and, therefore, are more likely to be younger, have more-complex symptoms, and possibly more education than the population at large. However, we do believe the results could be representative of a similar memory clinic population. The CDR-SB, although widely used, was not originally designed to measure progression, and we scored the CDR, post hoc, based on patient records. The ratings were, however, conducted by the same experienced and certified rater, thus ensuring consistency and reproducibility. Fifty-four percent of the patients did not have any registered comorbidity. Even though our population is quite young and has little comorbidity, we fear there is a risk of underreporting. However, since the number of comorbidities are low in all the groups, we expect this underreporting is evenly distributed. Lastly, we do not have information on concomitant incidences such as cerebrovascular events like stroke, or delirium during the follow-up period. Even though our population had little comorbidity at the time of diagnosis, such incidences could have accelerated the progression rate in some patients (Haaksma et al., 2017).

A strength of this study is that the sample was thoroughly examined, and all diagnoses were set according to research criteria based on an extensive examination protocol and biomarkers (most participants underwent MRI of the brain). Another advantage is the application of GBTM as a statistical tool in the present study. With GBTM, the trajectory groups are not based on ex ante characteristics; rather, it uses the actual variations in the data as a statistical tool to group those with similar development (Nagin and Odgers, 2010).

\section{Conclusion}

In a clinical cohort comprising patients with dementia, we identified three distinct trajectory groups of progression: one group with slow progression and two with more-rapid progression rates. Rapid progression was associated with older age, lower cognitive function, and more-pronounced NPS at the time of diagnosis. This knowledge can guide clinicians and policymakers in estimating prognoses and in planning for the future care of patients with dementia. Our findings also demonstrate the heterogeneity in dementia progression and the need for individual follow-up regimes. For future intervention studies, our results may guide the selection of patients and the follow-up period needed, since almost half of the patients progressed slowly up to 3 years after the diagnosis. Our results also show that, particularly in earlier stages of dementia, estimating prognoses can be difficult. Therefore, we need to search for other factors, such as biomarkers, that can predict the different trajectories of disease progression earlier in the disease process.

\section{Description of authors' roles}

Conceptualization: Knut Engedal, Geir Selbæk, Bjørn Heine Strand, and Anne-Brita Knapskog. Data curation: Trine Holt Edwin. Formal analysis: Trine Holt Edwin, Bjørn Heine Strand, and AnneBrita Knapskog. Investigation: Trine Holt Edwin, Anne-Brita Knapskog, and Karin Persson. Project administration: Geir Selbæk, Bjørn Heine Strand. Supervision: Anne-Brita Knapskog, Bjørn Heine Strand, and Geir Selbæk. Visualization: Trine Holt Edwin, Bjørn Heine Strand. Writing - original draft: Trine Holt Edwin, Bjørn Heine Strand, and Anne-Brita Knapskog. Writing - review and editing: Trine Holt Edwin, Bjørn Heine Strand, Karin Persson, Knut Engedal, Geir Selbæk, and Anne-Brita Knapskog.

\section{Conflict of interest}

Dr. Edwin, Dr. Knapskog, and Dr. Persson reports work with Roche BN29553 and with BoehringerIngelheim 1346.0023, outside the submitted work. The remaining authors have no conflicts of interest to declare.

\section{Source of funding}

This study was funded by the Norwegian Health Association, which was not involved in any part of 
the planning, conducting the study or writing the article. The authors have had full control of all the primary data. Legal restrictions, imposed by the registry owners and the ethical committee, prevent us from publicly sharing our de-identified data set due to sensitive patient information. Data may be requested from the Norwegian Register of Persons Assessed for Cognitive Symptoms (contact: post@aldringoghelse.no). Availability is dependent on approval from the Regional Ethics Committee for medical research in the SouthEast of Norway (contact: post@helseforsikring. etikkom.no).

\section{Acknowledgments}

This study was funded by the Norwegian Health Association, which was not involved in any part of planning and conducting the study or writing the paper. We would like to thank NorCog for their contribution to the study.

\section{Supplementary material}

To view supplementary material for this article, please visit https://doi.org/10.1017/S1041610220003270.

\section{References}

Blanc, F. et al. (2017). Long-term cognitive outcome of Alzheimer's disease and dementia with Lewy bodies: dual disease is worse. Alzheimer's Research and Therapy, 9, 47. doi: 10.1186/s13195-017-0272-8

Braekhus, A., Ulstein, I., Wyller, T. B. and Engedal, K. (2011). The Memory Clinic -outpatient assessment when dementia is suspected. Tidsskr Nor Laegeforen, 131, 2254-2257. doi: 10.4045/tidsskr.11.0786

Cummings, J. L., Mega, M., Gray, K., RosenbergThompson, S., Carusi, D. A. and Gornbein, J. (1994). The Neuropsychiatric Inventory: comprehensive assessment of psychopathology in dementia. Neurology, 44, 2308-2314.

Eldholm, R. S. et al. (2018a). Progression of Alzheimer's disease: a longitudinal study in Norwegian memory clinics. fournal of Alzheimer's Disease, 61, 1221-1232. doi: 10.3233/JAD-170436

Eldholm, R. S. et al. (2018b). Association between vascular comorbidity and progression of Alzheimer's disease: a twoyear observational study in Norwegian memory clinics. BMC Geriatrics, 18, 120. doi: 10.1186/s12877-018-0813-4

Emre, M. et al. (2007). Clinical diagnostic criteria for dementia associated with Parkinson's disease. Movement Disorders, 22, 1689-1707; quiz 1837. doi: 10.1002/ mds. 21507

Folstein, M. F., Folstein, S. E. and Mchugh, P. R. (1975). "Mini-mental state". A practical method for grading the cognitive state of patients for the clinician. Fournal of Psychiatric Research, 12, 189-198.

Gorno-Tempini, M. L. et al. (2011). Classification of primary progressive aphasia and its variants. Neurology, 76, 1006-1014. doi: 10.1212/WNL.0b013e31821103e6

Haaksma, M. L., Calderon-Larranaga, A., Olde Rikkert, M. G. M., Melis, R. J. F. and Leoutsakos, J. S. (2018). Cognitive and functional progression in Alzheimer disease: a prediction model of latent classes. International fournal of Geriatric Psychiatry, 33, 1057-1064. doi: $10.1002 /$ gps.4893

Haaksma, M. L. et al. (2017). Comorbidity and progression of late onset Alzheimer's disease: a systematic review. PLoS One, 12, e0177044. doi: 10.1371/journal.pone .0177044

Hughes, C. P., Berg, L., Danziger, W. L., Coben, L. A. and Martin, R. L. (1982). A new clinical scale for the staging of dementia. British fournal of Psychiatry, 140, 566-572.

Jones, B. L. and Nagin, D. S. (2013). A note on a stata plugin for estimating group-based trajectory models. Sociological Methods and Research, 42, 608-613. doi: 10.1177/0049124113503141

Jutkowitz, E., Maclehose, R. F., Gaugler, J. E., Dowd, B., Kuntz, K. M. and Kane, R. L. (2017). Risk factors associated with cognitive, functional, and behavioral trajectories of newly diagnosed dementia patients. Fournals of Gerontology Series A: Biological Sciences and Medical Sciences, 72, 251-258. doi: 10.1093/gerona/ glw079

Kaufer, D. I. et al. (2000). Validation of the NPI-Q, a brief clinical form of the Neuropsychiatric Inventory. Fournal of Neuropsychiatry and Clinical Neurosciences, 12, 233-239. doi: 10.1176/jnp.12.2.233

Knight ADRC. The Clinical Dementia rating (CDR) Online certification Training [Online]. Available at: http://alzheimer .wustl.edu/cdr/CDR.htm, last accessed 28 March 2017.

Krogseth, M. et al. (2016). Delirium is a risk factor for further cognitive decline in cognitively impaired hip fracture patients. Archives of Gerontology and Geriatrics, 64, 38-44. doi: 10.1016/j.archger.2015.12.004

Lanctot, K. L. et al. (2017). Neuropsychiatric signs and symptoms of Alzheimer's disease: new treatment paradigms. Alzheimers Dement (N Y), 3, 440-449. doi: 10.1016/ j.trci.2017.07.001

Lyketsos, C. G., Lopez, O., Jones, B., Fitzpatrick, A. L., Breitner, J. and Dekosky, S. (2002). Prevalence of neuropsychiatric symptoms in dementia and mild cognitive impairment: results from the cardiovascular health study. FAMA, 288, 1475-1483. doi: 10.1001/jama.288. 12.1475

Mainland, B. J., Amodeo, S. and Shulman, K. I. (2014). Multiple clock drawing scoring systems: simpler is better. International fournal of Geriatric Psychiatry, 29, 127-36. doi: 10.1002/gps.3992

Mckeith, I. G. et al. (2017). Diagnosis and management of dementia with Lewy bodies: fourth consensus report of the DLB consortium. Neurology, 89, 88-100. doi: 10.1212/ wnl.0000000000004058

Mckhann, G. M. et al. (2011). The diagnosis of dementia due to Alzheimer's disease: recommendations from the 
National Institute on Aging-Alzheimer's Association workgroups on diagnostic guidelines for Alzheimer's disease. Alzheimer's E Dementia, 7, 263-269. doi: 10.1016/ j.jalz.2011.03.005

Melis, R. J. F., Haaksma, M. L. and Muniz-Terrera, G. (2019). Understanding and predicting the longitudinal course of dementia. Current Opinion in Psychiatry, 32, 123-129. doi: 10.1097/yco.0000000000000482

Mitrushina, M., Nboone, K., Razani, L. and D'elia, L. (2005). Handbook of Normative Data for Neuropsychological Assessment, Oxford: Oxford University Press.

Morris, J. C. et al. (1989). The Consortium to Establish a Registry for Alzheimer's Disease (CERAD). Part I. Clinical and neuropsychological assessment of Alzheimer's disease. Neurology, 39, 1159-1165.

Musicco, M. et al. (2010). Neuropsychological predictors of rapidly progressing patients with Alzheimer's disease. Dementia and Geriatric Cognitive Disorders, 30, 219-228. doi: 10.1159/000319533

Nagin, D. S. and Odgers, C. L. (2010). Group-based trajectory modeling in clinical research. The Annual Review of Clinical Psychology, 6, 109-138. doi: 10.1146/ annurev.clinpsy.121208.131413

Norwegian Directorate of Health (2017). Nasjonal faglig retningslinje om demens. Oslo, Norway.

Norwegian Institute of Public Health. (2018). Cause of Death Registry [Online]. Bergen, Norway: Norwegian Institute of Public Health. Available at: https://www.fhi.no/ $\mathrm{hn} /$ helseregistre-og-registre/dodsarsaksregisteret/; last accessed 01 January 2018.

O'bryant, S. E. et al. (2008). Staging dementia using Clinical Dementia Rating Scale Sum of Boxes scores: a Texas Alzheimer's research consortium study. Archives of Neurology, 65, 1091-1095. doi: 10.1001/archneur.65. 8.1091

Poulin, S. P., Bergeron, D. and Dickerson, B. C. (2017). Risk factors, neuroanatomical correlates, and outcome of neuropsychiatric symptoms in Alzheimer's Disease.

Fournal of Alzheimer's Disease, 60, 483-493. doi: 10.3233/ jad-160767

Rascovsky, K. et al. (2011). Sensitivity of revised diagnostic criteria for the behavioural variant of frontotemporal dementia. Brain, 134, 2456-2477. doi: 10.1093/brain/ awr 179

Reed, C. et al. (2020). Factors associated with long-term impact on informal caregivers during Alzheimer's disease dementia progression: 36-month results from GERAS. International Psychogeriatrics, 32(2), 267-277. doi: 10.1017/ S1041610219000425

Reitan, R. M. (1958). Validity of the trail making test as an indicator of organic brain damage. Perceptual and Motor Skills, 8, 271-276.

Scheltens, N. M. E. et al. (2018). Prominent non-memory deficits in Alzheimer's disease are associated with faster disease progression. Fournal of Alzheimer's Disease, 65, 1029-1039. doi: 10.3233/JAD-171088

Schneider, L. S., Dagerman, K. S. and Insel, P. (2005). Risk of death with atypical antipsychotic drug treatment for dementia: meta-analysis of randomized placebocontrolled trials. FAMA, 294, 1934-1943. doi: 10.1001/ jama.294.15.1934

Shah, H. et al. (2016). Research priorities to reduce the global burden of dementia by 2025 . The Lancet Neurology, 15, 1285-1294. doi: 10.1016/s1474-4422(16)30235-6

Shulman, K., Shedletsky, R. and Silver, I. (1986). The challenge of time: clock-drawing and cognitive function in the elderly. International fournal of Geriatric Psychiatry, 1, 135-140. doi: 10.1002/gps.930010209

Siafarikas, N., Selbaek, G., Fladby, T., Saltyte Benth, J., Auning, E. and Aarsland, D. (2018). Frequency and subgroups of neuropsychiatric symptoms in mild cognitive impairment and different stages of dementia in Alzheimer's disease. International Psychogeriatrics, 30, 103-113. doi: $10.1017 / \mathrm{s} 1041610217001879$

Song, Y. N. et al. (2018). Risk factors of rapid cognitive decline in Alzheimer's disease and mild cognitive impairment: a systematic review and meta-analysis. Fournal of Alzheimer's Disease, 66, 497-515. doi: 10.3233/ jad-180476

Strand, B. H. et al. (2018). Survival and years of life lost in various aetiologies of dementia, mild cognitive impairment (MCI) and subjective cognitive decline (SCD) in Norway. PLoS One, 13, e0204436. doi: 10.1371/journal.pone. 0204436

Strand, B. H. et al. (2019). The Loss in Expectation of Life due to Early-Onset Mild Cognitive Impairment and EarlyOnset Dementia in Norway. Dementia and Geriatric Cognitive Disorders, 47, 355-365. doi: 10.1159/000501269

Tschanz, J. T. et al. (2011). Progression of cognitive, functional, and neuropsychiatric symptom domains in a population cohort with Alzheimer dementia: the Cache County Dementia Progression study. The American fournal of Geriatric Psychiatry, 19, 532-542. doi: 10.1097/ JGP.0b013e3181faec23

Vasilevskis, E. E., Han, J. H., Hughes, C. G. and Ely, E. W. (2012). Epidemiology and risk factors for delirium across hospital settings. Best Practice and Research: Clinical Anaesthesiology, 26, 277-287. doi: 10.1016/j.bpa.2012. 07.003

Wang, Y. et al. (2019). Cognitive and functional progression of dementia in two longitudinal studies. International fournal of Geriatric Psychiatry, 34, 1623-1632. doi: 10.1002/ gps.5175

Xu, J., Murphy, S. L., Kochanek, K. D., Bastian, B. and Arias, E. (2018). Deaths: Final Data for 2016. National vital statistics reports: from the Centers for Disease Control and Prevention, National Center for Health Statistics. National Vital Statistics System, 67, 1-76. 